\title{
Plateau pressure during pressure control ventilation
}

\author{
Simone Sosio ${ }^{1}$, Giacomo Bellani ${ }^{1,2}$ \\ ${ }^{1}$ Department of Medicine and Surgery, University of Milan-Bicocca, Monza - Italy \\ ${ }^{2}$ Department of Emergency and Intensive Care, San Gerardo Hospital, Monza - Italy
}

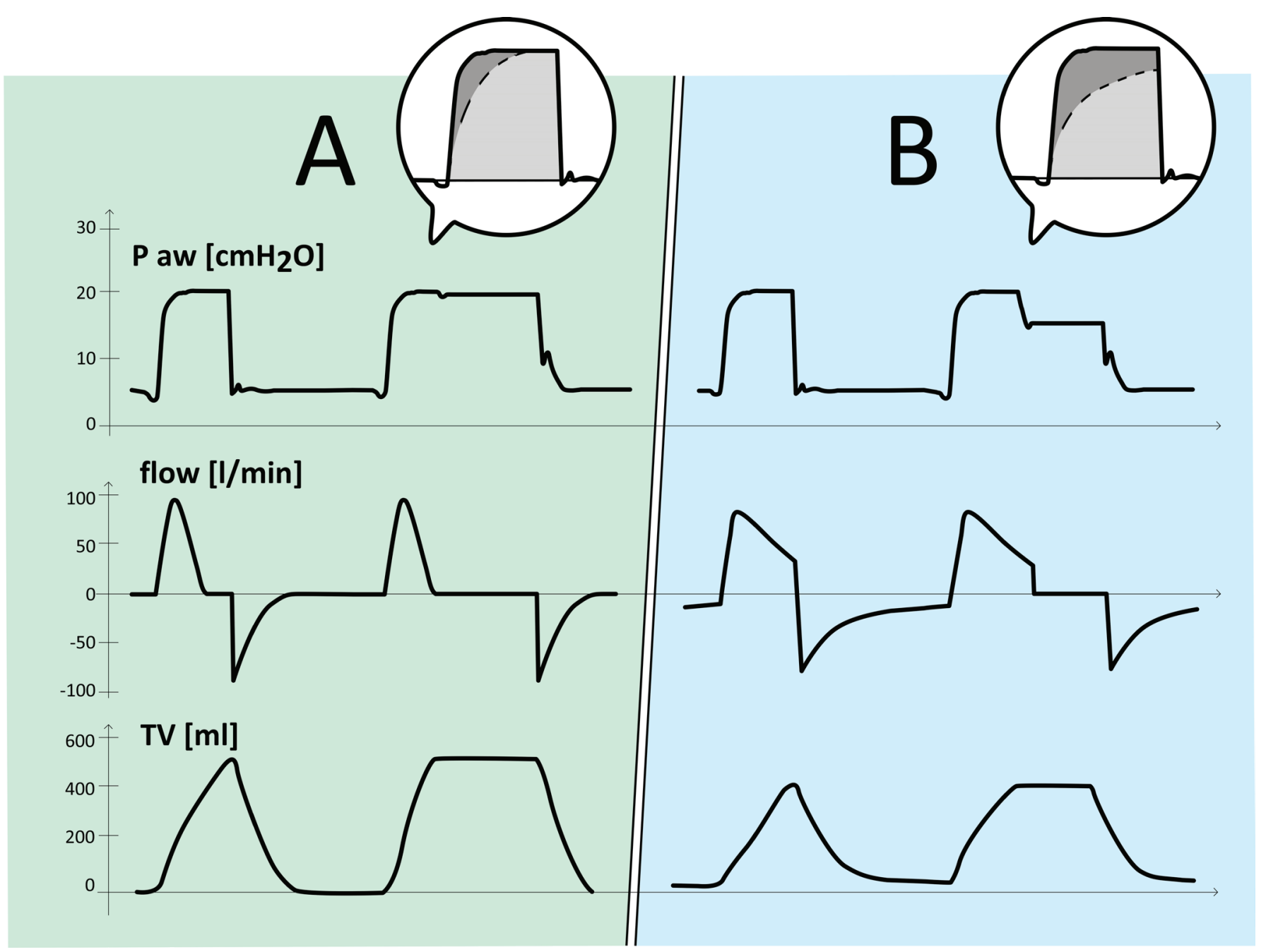

Submitted: September 3, 2019

Accepted: September 19, 2019

Published online: October 22, 2019

\section{Corresponding author}

Giacomo Bellani

Department of Medicine and Surgery

University of Milan-Bicocca

Via Cadore 48

Monza (MB), Italy

giacomo.bellani1@unimib.it

\section{Image description}

The airway pressure normally seen on the ventilator screen is the total pressure applied on the respiratory system. It is the sum of the elastic and the resistive pressures. In the balloons this is shown in detail: the pressure that inflates the alveoli is shown in light gray, the pressure that is spent to generate the flow is shown in dark gray. The pressure applied to the alveoli, net of the resistive pressure, can be revealed by an inspiratory pause: once the flow reduces to zero, only the elastic pressure distending the lung is seen. This is the correct 
Plateau pressure (Pplat). In subject $A$, due to low resistance, flow reaches zero during normal respiratory cycles also, so that Ppeak coincides with Pplat. In subject $B$, due to resistive load, flow does not reach zero value at the end of inspiration and Pplat (revealed by inspiratory hold) is lower than Ppeak.

\section{Discussion}

During mechanical ventilation, the plateau pressure (Pplat) is the pressure applied at the end of inspiration to small airways and alveoli and it is measured during an inspiratory pause (or hold) on the ventilator. It represents the pressure generated, in the alveoli, by the elastic recoil of the respiratory system. Both driving pressure (DP) and respiratory system compliance ( $\mathrm{Cpl}, \mathrm{rs})$ calculation include Pplat. The absence of flow during the measurement is crucial, since it allows equilibration of the pressure in the alveoli and in the ventilator.

It is accepted that the estimation of both Pplat and DP during passive, controlled ventilation is a key element to provide a protective ventilation (1). Moreover, the estimation of Pplat and DP during pressure support, in the presence of spontaneous breathing, has been recently proposed (2).

However, the measurement of Pplat (and DP and Cpl,rs) in pressure control ventilation (PCV) is often a source of confusion, even in experienced clinicians: a common misbelief is that, inspiratory pressure (Pinsp) during PCV having a flat shape, this represents a Pplat.

Before expanding the concept of Pplat measurement during PCV, we need to resume a specific concept. The ventilator, in order to deliver the tidal volume, must apply a positive pressure (a) to overcome the resistances to the airflow in the airways (resistive pressure, not exerted on the alveoli) and (b) to inflate the alveoli (elastic pressure) (3). The pressure generated by the ventilator during inspiration $(a+b)$ is the peak pressure (Ppeak), sometimes referred to as Pinsp in PCV. The heterogeneity of nomenclature used by ventilator brands, unfortunately, adds to confusion (4).

Depending on the airway resistance and the inspiratory time, the flow might get to zero at the end of inspiration. Hence Pplat will be similar to Ppeak or Pinsp (Fig. 1, subject A).
On the contrary, in the presence of resistive load and/or short inspiratory time, flow will still be present at the end of inspiration and a manual inspiratory hold is required to measure Pplat. In these conditions, following an inspiratory hold, the pressure curve will decrease and equilibrate with the alveolar pressure, so Pplat will be less than Ppeak or Pinsp (Fig. 1, subject B).

\section{Conclusion}

In conclusion during PCV the Ppeak (albeit flat in shape) is not reliable for the purpose of a protective ventilation in a critical patient. Conversely, an inspiratory hold in PCV allows to estimate the Pplat, that is, the actual and inspiratory alveolar pressure (and hence to calculate correctly the DP and Cpl,rs).

\section{Acknowledgment}

The authors thank Davide De Ponti, University of Milan, Bicocca, for his help in manuscript preparation.

\section{Disclosures}

Financial support: Departmental funding.

Conflict of interest: Authors have no conflict of interest to declare in relation to the present manuscript.

\section{References}

1. Amato MBP, Meade MO, Slutsky AS, et al. Driving pressure and survival in the acute respiratory distress syndrome. N Engl J Med. 2015 Feb 19;372(8):747-755.

2. Bellani G, Grassi A, Sosio S, Foti G. Plateau and driving pressure in the presence of spontaneous breathing. Intensive Care Med. 2019;45:97-98.

3. Brander L, Slutsky AS. Invasive mechanical ventilation in: Spiro S, Silvestri G, Agustí A. Clinical Respiratory Medicine 4th ed.: Elsevier; 2012: 406-430.

4. Mireles-Cabodevila E, Hatipoğlu U, Chatburn RL. A rational framework for selecting modes of ventilation. Respir Care. 2013;58:348-366. 